\title{
REFERENCE SURFACES AND GLOBAL FIGURE PARAMETERS OF THE TERRESTRIAL PLANETARY BODIES
}

\author{
K.K. KAMENSKY \\ SKTB of Institute for Applied Problems of Mechanics and Mathematics \\ Ul. Lermontova 15 \\ 290005 Lvov \\ USSR
}

For connecting the inertial coordinate system with the non-inertial planetocentric one we need to know values of the fundamental astronomical constants as well as global figure parameters of the planet. We distinguish among planetary figures a dynamical, a level, and hypsometric figures.

Parameters of the last two figures can be determined by using the least squares method under the condition:

$$
\int_{0}^{2 \pi} \int_{-\frac{\pi}{2}}^{+\frac{\pi}{2}} p(\varphi, \lambda)\left[r_{e}\left(\varphi, \lambda, a, b, c, \lambda_{a}, \varphi_{c}, \lambda_{c}\right)-r(\varphi, \lambda)\right]^{2} d \varphi d \lambda=\min
$$

where $p$ is weighting function averaging for the trapezium with the centre coordinates $\varphi, \lambda ; r$ and $r_{e}$ are the corresponding mean values of the radii of the surface being approximated, and of the approximating free-oriented triaxial ellipsoid with semiaxes $a>b>c$, and three orienting angles, for instance, $\lambda_{\mathbf{z}}, \varphi_{\mathrm{c}}, \lambda_{\mathrm{c}}$.

The results of computations (see the table) show that orientation of the level figures is very close to the orientation of the dynamical ones, determined by other authors using different methods. Flattenings of these figures are also near to the values recommended by the IAU as the reference surfaces parameters [1]. The smallest axes are close to the rotation axes for the planets with fast axial rotation and pronounced biaxiality (e.g., the Earth and Mars). The angle between the axes of figure and of rotation for Venus reaches the value of $3: 6$. Using other data we have obtained an even larger value of $11^{\circ}[2]$.

In an inertial frame of reference a level surface becomes an equipotential surface of attractive potential because there is no inertial force and other fictitious forces in this frame. Numerical investigations of this phenomenon were conducted in [3]. Analysis of the results obtained shows that in the case of the Earth and Mars the flattening of the attractive potential figure is about two times greater than for the gravity potential figure. The axis of the former figure is closer to the rotation axis than for the latter one. 
The smallest axes of hypsometric figure (see numerical quantities in [3]) diverge considerably from the rotational axes and the inertia axes of the planets. At the same time, flattenings of hypsometric surfaces are much closer to flattenings of attractive potential figures than of gravity ones.

The Earth's level figures investigation shows different orientation of the smallest axis for different geopotential models. The last circumstance can be explained by the complex motion of the terrestrial coordinate system relative to the inertial one and by the discrepancy of geopotential models.

Table. Global gravity figure parameters of the terrestrial planets and the Moon

\begin{tabular}{llllll} 
Parameter & Dimension & Venus & Earth & Moon & Mars \\
\hline & & & & & \\
$G M$ & $10^{9} \mathrm{~m}^{3} / \mathrm{s}^{2}$ & 324858.15 & 398600.448 & 4902.799 & 42828.44 \\
$\omega$ & $10^{5} \mathrm{rad} / \mathrm{s}$ & 0.029924 & 7.292115 & 0.266170 & 7.087919 \\
$a$ & $\mathrm{~m}$ & 6051473. & 6370831. & 1737861. & 3391212. \\
$b$ & $\mathrm{~m}$ & 6051448. & 6370762. & 1737630. & 3389929. \\
$c$ & $\mathrm{~m}$ & 6051422. & 6349458. & 1737212. & 3372848. \\
$\lambda_{\mathrm{a}}$ & $\mathrm{deg}$ & $353.5 \mathrm{E}$ & $345.0576 \mathrm{E}$ & $0.02585 \mathrm{E}$ & $104.905 \mathrm{~W}$ \\
$\varphi_{\mathrm{c}}$ & $\mathrm{deg}$ & $86.4 \mathrm{~N}$ & $90.0000 \mathrm{~N}$ & $89.98000 \mathrm{~N}$ & $89.999 \mathrm{~N}$ \\
$\lambda_{\mathrm{c}}$ & $\mathrm{deg}$ & $75.4 \mathrm{E}$ & $69.2912 \mathrm{E}$ & $0.88594 \mathrm{E}$ & $195.634 \mathrm{~W}$
\end{tabular}

\section{References}

1. Davies, M., Abalakin, V.K., Burša, M. et al. (1986) "Report of the IAU/IAG/COSPAR working group on cartographic coordinates and rotational elements of the planets and satellites, 1985", Celestial Mechanics 39,103-113.

2. Kamensky, K.K. (1988) "The research of global pecularities of Venus equipotential and hypsometric surfaces", in Ya.S. Yatskiv (ed.), The study of the Earth as a planet by methods of geophysics, geodesy and astronomy, Naukova Dumka, Kiev, pp. 80-83, (in Russian).

3. Kamensky, K.K., Kislyuk, V.S., Yatskiv, Ya.S. (1988) "Geometrical and dynamical characteristics of the Earth, the Moon and the terrestrial planets: 1. Topographic surfaces and gravitational fields", Preprint Int. Theoretical Physics ИТФ-88-86Р, Kiev, (in Russian). 